\title{
Endothelin inhibitors for advanced prostate cancer (Protocol)
}

\author{
Foley R, Keane J, O'Mathúna DP, Hollywood D
}
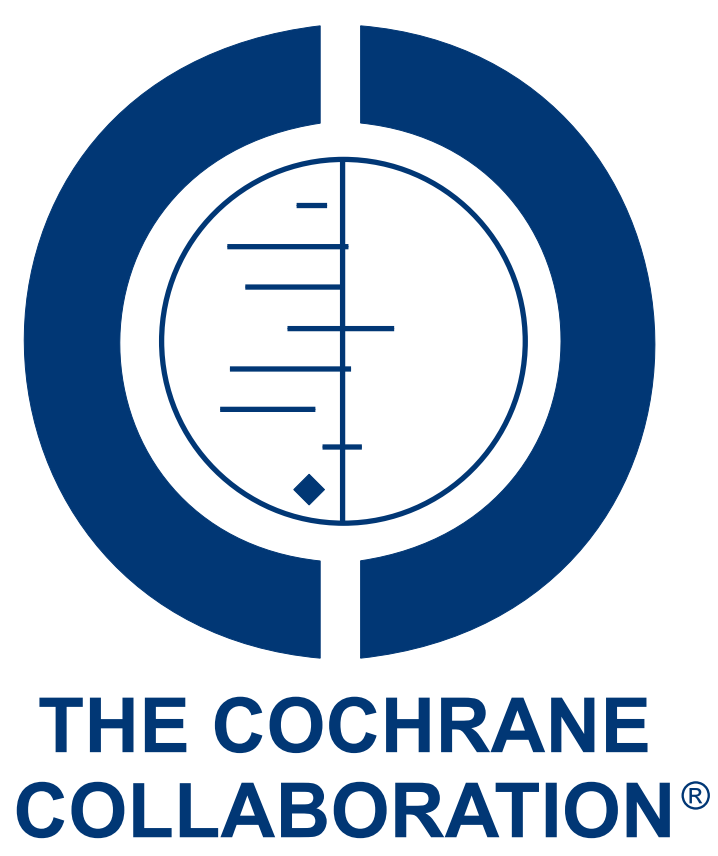

This is a reprint of a Cochrane protocol, prepared and maintained by The Cochrane Collaboration and published in The Cochrane Library 2011, Issue 8

http://www.thecochranelibrary.com

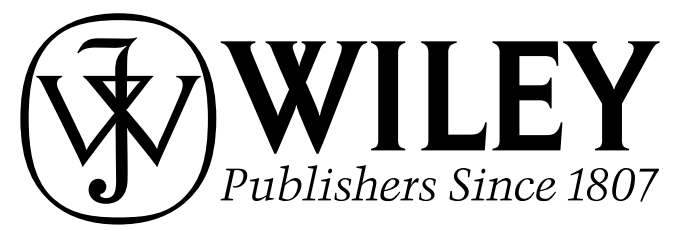

Endothelin inhibitors for advanced prostate cancer (Protocol)

Copyright $\odot 2011$ The Cochrane Collaboration. Published by John Wiley \& Sons, Ltd. 


\section{TABLE OF CONTENTS}

HEADER . . . . . . . . . . . . . . . . . . . . . . . . . . . . . . . . . . . . . . . 1

ABSTRACT . . . . . . . . . . . . . . . . . . . . . . . . . . . . . . . . . . . . . . . 1

BACKGROUND . . . . . . . . . . . . . . . . . . . . . . . . . . . . . . . . . . 2

OBJECTIVES . . . . . . . . . . . . . . . . . . . . . . . . . . . . . . . . . . . . . .

METHODS . . . . . . . . . . . . . . . . . . . . . . . . . . . . . . . . . . . . . . .

ACKNOWLEDGEMENTS . . . . . . . . . . . . . . . . . . . . . . . . . . . . . . . . . . . . . . .

REFERENCES . . . . . . . . . . . . . . . . . . . . . . . . . . . . . . . . . . . . . . 6

APPENDICES . . . . . . . . . . . . . . . . . . . . . . . . . . . . . . . . . . . . . 8

HISTORY . . . . . . . . . . . . . . . . . . . . . . . . . . . . . . . . . . . . . . . 8

CONTRIBUTIONS OF AUTHORS . . . . . . . . . . . . . . . . . . . . . . . . . . . . . . . . . . . . . . . . . .

DECLARATIONS OF INTEREST . . . . . . . . . . . . . . . . . . . . . . . . . . . . . . . . . . . . . . .

SOURCES OF SUPPORT . . . . . . . . . . . . . . . . . . . . . . . . . . . . . . . . . . . . . . . . . . 


\title{
[Intervention Protocol]
}

\section{Endothelin inhibitors for advanced prostate cancer}

\author{
Ruth Foley ${ }^{1}$, John Keane ${ }^{2}$, Dónal P O’Mathúna ${ }^{3}$, Donal Hollywood ${ }^{4}$ \\ ${ }^{1}$ Academic Unit of Clinical and Molecular Oncology, School of Medicine, Trinity College Dublin, Dublin, Ireland. ${ }^{2}$ Altnagelvin Area \\ Hospital, Derry, UK. ${ }^{3}$ School of Nursing, Dublin City University, Dublin, Ireland. ${ }^{4}$ Division of Radiation Therapy, Trinity Centre for \\ Health Sciences, St. James' Hospital, Dublin, Ireland \\ Contact address: Ruth Foley, Academic Unit of Clinical and Molecular Oncology, School of Medicine, Trinity College Dublin, Dublin, \\ Ireland. ruth.foley@tcd.ie.ruthfoley2010@gmail.com.
}

Editorial group: Cochrane Prostatic Diseases and Urologic Cancers Group.

Publication status and date: New, published in Issue 8, 2011.

Citation: Foley R, Keane J, O'Mathúna DP, Hollywood D. Endothelin inhibitors for advanced prostate cancer. Cochrane Database of Systematic Reviews 2011, Issue 8. Art. No.: CD009193. DOI: 10.1002/14651858.CD009193.

Copyright (C) 2011 The Cochrane Collaboration. Published by John Wiley \& Sons, Ltd.

\section{A B S T R A C T}

This is the protocol for a review and there is no abstract. The objectives are as follows:

Our review aims to determine the effectiveness and adverse effects of endothelin inhibitors for advanced prostate cancer.

The primary objectives are to assess the effect of endothelin inhibitors on overall survival, prostate cancer-specific survival, and progression-free survival.

The secondary objectives are to determine:

1. the frequency of adverse events;

2. the effect of treatment on target lesions identified by radiological imaging;

3. the effect of treatment on molecular biomarkers, including PSA and bone alkaline phosphatase;

4. the impact on quality of life. 


\section{B A C K G ROU N D}

\section{Description of the condition}

Prostate cancer is the second most frequently diagnosed cancer, and the sixth most common fatal cancer, in men worldwide. It is most common in developed countries, but up to 50 times less frequently diagnosed in Asian countries. The peak incidence of 179 per 100,000 occurs in the USA's black population, in which the mortality rate is 26 per 100,000 (Jemal 2010). Prostate cancer is rarely diagnosed before the age of 50, but becomes more common with increasing age (Kirby 2001). While many prostate tumours grow slowly and with little effect on life expectancy, advanced prostate cancer significantly limits both survival and quality of life. Prostate cancer is definitively diagnosed by histological examination of transrectal ultrasound-guided biopsy samples of prostate tissue. Presenting features may include bladder outflow symptoms, urinary tract problems, and in more advanced presentations, pain due to metastatic disease. Most cancers originate in the periphery of the gland and do not cause symptoms in the early stage of the disease. The majority of men are asymptomatic and undergo biopsy following detection of an abnormal gland on digital rectal examination or by the presence of an abnormal level of serum prostate-specific antigen (PSA), a simple blood test. Widespread PSA testing has resulted in the diagnosis of many clinically insignificant tumours, and a meta-analysis of randomised controlled trials concluded that screening did not significantly reduce diseasespecific mortality (Ilic 2010).

The majority of patients present with localised or locally advanced disease, which is potentially curable by radical surgery or radical radiotherapy with neoadjuvant androgen deprivation therapy (ADT). This review focuses on a later stage of prostate cancer progression, when the disease has become metastatic and/or castration-resistant. These clinical entities account for most of the morbidity and mortality caused by prostate cancer.

Metastatic disease is confirmed when a bone scan, computed tomography or magnetic resonance imaging shows secondary spread of prostatic adenocarcinoma beyond the prostate gland and pelvic lymph nodes. Prostate cancer frequently metastasises to bone (Catalona 1984), which can give rise to debilitating pain, spinal cord compression and pathological fractures. Metastatic prostate cancer is currently incurable and has a 5-year survival of approximately $25 \%$, while less than $10 \%$ of patients survive beyond 10 years (Tangen 2003).

Locally advanced, metastatic and recurrent prostate cancer is highly sensitive to hormonal manipulation by ADT using luteinising hormone-releasing hormone analogues to block testosterone production. Response to treatment can be accurately assessed by serum PSA levels. However, given a sufficient time period on ADT, the disease will become resistant and recur, heralded by a rising PSA. This terminal phase of the disease is known as castrationresistant prostate cancer.
The terms "hormone-refractory prostate cancer" and "androgenindependent prostate cancer" have also been used to describe this phase, but are misleading for a variety of reasons, such as the fact that the androgen biosynthesis inhibitor, abiraterone acetate, has recently been shown to improve overall survival in castration resistant prostate cancer (de Bono 2010). We will therefore use the more appropriate term "castration-resistant prostate cancer". At this stage, disease symptoms increase, biochemical progression defined by rising serum PSA levels occurs, or there is radiological evidence of metastatic tumour growth (NICE 2008). In patients with metastatic disease, biochemical progression occurs after a median of 24 months of treatment with ADT (Benaim 2002). Median survival after development of castration-resistant prostate cancer has recently been reported to be 40 months in the presence of confirmed bone metastases, and 68 months when bone metastases are absent (Oefelein 2004).

Serum PSA is a very useful indicator of the success or failure of conventional therapy, though optimal cutoff values are a matter of discussion (Scher 2004). Importantly, PSA responses are not always reliable indicators of the efficacy of experimental therapies (Dahut 2008; Nabhan 2009). As a result, the Prostate Cancer Clinical Trials Working Group (PCWG2) has recommended that PSA should not be used as the sole criterion for identifying disease progression and therefore stopping treatment with a trial drug (Scher 2008). The PCWG2 also provided guidelines to address uncertainty on how to define response to therapy using bone scans (Scher 2008).

\section{Description of the intervention}

The endothelin molecular pathway was discovered in 1988 while searching for substances which widen or narrow blood vessels (Yanagisawa 1988). This pathway includes several molecules involved in the regulation of multiple aspects of cell and tissue function, including cell division, cell death and blood vessel organisation (Nelson 2003). To activate the pathway, a short peptide such as endothelin-1 (ET-1) binds to a cell surface protein such as endothelin $\mathrm{A}\left(\mathrm{ET}_{A}\right)$. The cell surface receptor then interacts directly or indirectly with multiple molecules within the cell to influence its behaviour and interaction with other cells. ET-1 has been extensively studied as a potent agent for constricting blood vessel diameter (Battistini 2006), and has also been investigated in the last decade as a target for cancer therapy (Russo 2010). Atrasentan (Xinlay ${ }^{T M}$, ABT-627) is a highly potent and selective $\mathrm{ET}_{A}$ receptor antagonist. In humans, atrasentan achieves physiologically active plasma concentrations when administered orally, once daily (Carducci 2002). Multi-dose pharmacokinetics are predictable with atrasentan. Maximal plasma concentrations are typically achieved within 1.5 hours and the half-life is approximately 24 hours (Carducci 2002). The maximum tolerated dose of atrasentan appears to be $60 \mathrm{mg}$, but most clinical studies use doses no greater than $30 \mathrm{mg}$ to avoid non-specific toxicities, while 
the most common side effects reported are rhinitis, headache, asthenia and peripheral oedema (Carducci 2002).

Zibotentan (ZD4054) is another selective $\mathrm{ET}_{A}$ receptor antagonist that blocks or reverses the biologic effects of ET-1. In humans, zibotentan achieves physiologically active plasma concentrations when administered orally, once daily. Multi-dose pharmacokinetics are predictable with zibotentan. The maximal plasma concentration is typically achieved within 3 hours of dosing and the halflife is approximately 12 hours (Ranson 2010). No dose-limiting toxicity was observed up to $15 \mathrm{mg}$ per day (Trump 2010). It is generally well tolerated with the most common reported side-effects being headache, peripheral oedema, fatigue, nasal congestion and nausea (Schelman 2011).

Treatment options for patients with advanced prostate cancer are currently limited. In addition to hormonal therapy, chemotherapy using docetaxel, mitoxantrone and prednisone have been used in castration-resistant disease and may improve survival and management of symptoms, but the treatment is associated with significant toxicity, particularly myelosuppression, gastrointestinal toxicity, cardiac toxicity, neuropathy, and alopecia (Shelley 2006).

\section{How the intervention might work}

The endothelin pathway stimulates tumour growth by increasing cell proliferation directly and in co-operation with other growth factors, decreasing cell death, and enhancing the formation of new blood vessels (angiogenesis) (Nelson 2003). These properties prompted the development of drugs blocking this pathway as a novel strategy for cancer treatment.

Several aspects of endothelin biochemistry support this pathway as a particularly attractive target in prostate cancer patients with bone metastases. For example, ET-1 promotes the formation of osteoblastic bone metastases in an in vivo breast cancer model, similar to those observed in prostate cancer bone lesions (Yin 2003). Prostate epithelial cells express abundant ET1 (Langenstroer 1993), and its production is further increased by transforming growth factor beta (Le Brun 1999) produced in bone (Robey 1987). Thus a positive feedback loop may exist between ET-1 expression and osteoblast proliferation, contributing to the occurrence of bone metastases in prostate cancer patients. In addition, ET-1 has been associated with pain responses and may be involved in bone pain (Yuyama 2004).

Interestingly, a number of therapies targeted to other molecular pathways have been shown to be effective against other cancers. For example, trastuzumab, which blocks the HER2 growth factor pathway, has been developed and approved over the last two decades for breast cancer treatment, although other targeted therapies have been less successful (Normanno 2009).

\section{Why it is important to do this review}

Current therapeutic options for advanced hormone-dependent and castration-resistant prostate cancer are limited in their efficacy and significantly affect patient quality of life. Numerous clinical trials are seeking to overcome these challenges and bring forward new or improved therapeutic regimens. For example, in the USA, over fifty clinical trials of chemotherapy, targeted therapies and immunotherapy for metastatic prostate cancer are currently in progress (National Library of Medicine (US) 2011).

This review will provide a systematic analysis of the benefits and disadvantages of endothelin inhibitor treatment for advanced prostate cancer, and therefore contribute to facilitating evidencebased treatment decisions in a complex field with vast amounts of emerging clinical data. We will also identify questions which remain to be addressed as priorities in designing future clinical trials.

\section{O B JE C T I VES}

Our review aims to determine the effectiveness and adverse effects of endothelin inhibitors for advanced prostate cancer.

The primary objectives are to assess the effect of endothelin inhibitors on overall survival, prostate cancer-specific survival, and progression-free survival.

The secondary objectives are to determine:

1. the frequency of adverse events;

2. the effect of treatment on target lesions identified by radiological imaging;

3. the effect of treatment on molecular biomarkers, including PSA and bone alkaline phosphatase;

4. the impact on quality of life.

\section{METHODS}

\section{Criteria for considering studies for this review}

\section{Types of studies}

Only randomised controlled trials will be eligible. We will not accept quasi-randomised studies for inclusion. 


\section{Types of participants}

This review will consider studies of prostate cancer patients with either castration-resistant disease, metastatic disease, or both. Castration-resistant patients will have experienced clinical disease progression, radiological disease progression or PSA recurrence during hormone treatment. Eligible studies may include patients with symptomatic or asymptomatic metastases, patients who have received no prior therapy for prostate cancer, or patients who have been previously treated by interventions other than endothelin inhibitors.

\section{Types of interventions}

Patients will be treated with endothelin inhibitor drugs. This category of drugs includes but is not restricted to atrasentan and zibotentan. Drugs for which experimental evidence has been published, documenting statistically significant inhibition of the endothelin signalling pathway, will also be included.

Studies will be included which compare a defined endothelin inhibitor treatment regimen to placebo, to treatment with another drug including a different endothelin inhibitor, or to a different dose or duration of treatment with the same endothelin inhibitor. Concurrent treatment with one or more other therapies will be acceptable.

\section{Types of outcome measures}

We will include studies which report one or more of the following outcomes.

\section{Primary outcomes}

The review will examine overall survival, prostate cancer-specific survival, progression-free survival and time to progression as primary outcomes.

\section{Secondary outcomes}

1. We will analyse the proportion of patients who have experienced adverse events. In addition, we will analyse the frequency of clinically relevant events subdivided into:

$\diamond$ serious adverse events (Grades 3 and 4);

$\diamond$ metastatic events to include spinal cord

compression and pathological fractures;

$\diamond$ local progression events to include acute urinary retention and post-obstructive renal failure;

$\diamond$ systemic effects of treatment to include motor or sensory neuropathy.

2. We will examine the response to treatment of radiologically identified lesions, based on the Response Evaluation Criteria in Solid Tumours (RECIST) guidelines (see 'Appendix 1'). These guidelines were produced by an international collaborative task force, and utilise a wide range of clinical, radiological and invasive visualisation parameters for tumour response evaluation (Eisenhauer 2009).

3. Changes in biomarker concentrations during treatment will be analysed. While increases or decreases in serum PSA levels are not necessarily indicative of the effectiveness of experimental therapies, PSA is the best established serum biomarker in prostate cancer, and there is significant public awareness and interest in PSA testing in some countries. The PSA Working Group (PSAWG), through a consensus conference, developed guidelines for the use of PSA as a measurement outcome in phase II therapeutic trials. They advised that PSA response should be defined as a PSA decline of $50 \%$ or more, to be confirmed by a second PSA test at least four weeks later, with no clinical or radiographic evidence of disease progression (Bubley 1999). The PSAWG also defined biochemical progression as post-treatment increase of $50 \%$ from nadir with a PSA level of at least $5 \mathrm{ng} / \mathrm{mL}$ (nanograms/millilitre). Where data are available, we will analyse the frequency of PSA response, and of biochemical progression. Levels of bone alkaline phosphatase in serum will be analysed as the change in concentration following treatment, in addition to other molecular or cellular markers in serum or urine which are reported.

4. Quality of life and health-related quality of life will be reported.

\section{Search methods for identification of studies}

\section{Electronic searches}

The following literature databases will be searched: the Cochrane Central Register of Controlled Trials (CENTRAL), MEDLINE, EMBASE, LILACS, Science Citation Index Expanded, BIOSIS and the Australasian Medical Index. The Related Articles function will be used where available from eligible articles. Reports in any language will be considered and translations will be obtained of relevant studies. We will search databases from 1988 as the endothelin pathway was not studied before this date (Yanagisawa 1988).

MEDLINE will be searched through PubMed for the exploded $\mathrm{MeSH}$ terms "prostatic neoplasms" and endothelin, with a filter for randomised trials, and the text terms atrasentan, Xinlay, ABT627, ZD4054, and zibotentan. Search terms will be modified as required for other databases and sources.

The registers of clinical trials listed below will be searched.

http://www.controlled-trials.com

http://www.clinicaltrials.gov

http://www.eortc.be

http://www.who.int/ictrp/en/

http://www.clinicalstudyresults.org/ 


\section{Searching other resources}

Conference proceedings for the American Society of Clinical Oncology, European CanCer Organisation ${ }^{\circledR}$ (ECCO), European Society for Medical Oncology, European Association of Urology and American Urological Association, will be handsearched from 2000. We selected this date as Carducci 2002 reported the first use of endothelin inhibitors in cancer patients and earlier records are unlikely to yield eligible studies. Experts in the field and drug manufacturers will be contacted to enquire whether relevant trials or articles exist which are not listed in other sources. We will also examine the reference lists of obtained articles, systematic reviews, and clinical practice guidelines to check for other related published and unpublished studies.

\section{Data collection and analysis}

\section{Selection of studies}

Search results will be collected and reports that are clearly ineligible, or duplicate copies, will be removed based on titles and abstracts. We will retrieve the full text of the remaining reports. Two authors (RF and JK) will independently assess each study for eligibility using a standardised form. Study authors will be contacted, if necessary, for further information. Disagreements will be resolved by discussion and if necessary by reference to a third author.

Conference proceedings will be included if enough data is available, either in the abstract or through correspondence with the authors, to analyse one or more of the specified outcomes.

\section{Data extraction and management}

We will extract data on study design, participants, tumour characteristics, interventions, controls, outcomes, and information required to assess risk of bias. We will use a data extraction form designed for this review and tested in a pilot data collection exercise. Data will be extracted independently in duplicate by two authors (RF and JK). Disagreements will be resolved by discussion and if necessary by reference to a third author.

\section{Assessment of risk of bias in included studies}

We will use The Cochrane Collaboration's tool for assessing risk of bias as outlined in the Chapter 8 of the Cochrane Handbook (Higgins 2011). We will use the domain-based analysis tool for assessing risk of bias as presented in the Handbook's Table 8.5a. Studies will be assessed in terms of selection bias (randomisation), performance bias (blinding), attrition bias, detection bias, reporting bias and any other potential sources of bias. The data will be presented as low risk, high risk or unclear risk of bias as recommended in the Handbook guidelines. If the risk ratio of adverse events varies over time during follow up, and if such variation shows that the treatment effect could have been significantly influenced by the points at which the data was censored, this will be judged as a high risk of bias, in the "detection bias" category.

\section{Measures of treatment effect}

Overall survival, disease-specific survival, and progression-free survival will be measured firstly as hazard ratios and entered as generic inverse variance data. In addition, the percentage of patients surviving at a given time point will be analysed as dichotomous data using risk ratios, assuming that survival status is known for all patients at this time point. Time to progression will be measured as a continuous outcome using difference between means, with median rather than mean values if the data does not form a normal distribution, but not used for meta-analysis.

Adverse events, PSA response and biochemical progression will be analysed as dichotomous outcomes using risk ratios. Serum or urine concentrations of other biomarkers, where available, will be measured as continuous outcomes using difference between means. Quality of life measures will be based on subjective scales and summarised as standardised mean difference.

Tumour response to treatment, reported according to RECIST criteria, will be treated as ordinal data with four categories (Appendix 1) and analysed as follows. As the effect measure in each trial, we will calculate the log odds ratio by proportional odds logistic regression, using SPSS software. The log odds ratio will then be entered in RevMan as generic inverse variance data. The proportional odds model assumes that, when ordinal categories are converted to dichotomous data, the resulting odds ratios are similar regardless of how the categories are divided in two. If this assumption is not upheld, we will not perform logistic regression. Instead, we will analyse RECIST outcomes as dichotomous data using risk ratios, for partial and stable responses versus progressive disease. It is not expected that any complete responses to treatment will be observed, but progressive disease versus all other categories will be included if complete responses were to occur.

The inverse variance analysis method will be used. Dichotomous and categorical outcomes will be expressed with 95\% CI (confidence intervals). Intent-to-treat analyses will be used where possible and noted when not.

\section{Unit of analysis issues}

For the primary outcomes of overall survival, disease-specific survival, and progression-free survival, which may be reported at various time points such as 1,2 or 5 years, all available time points for which enough data is available for meta-analysis will be analysed.

\section{Dealing with missing data}

Study authors will be contacted to request relevant data which may have been collected but was not published. 


\section{Assessment of heterogeneity}

We will assess heterogeneity by visual inspection of forest plots and based on the $\mathrm{I}^{2}$ statistic generated by RevMan.

\section{Assessment of reporting biases}

For studies for which both protocol information and outcome data are available, a table will be generated to compare the outcomes which the studies planned to analyse, and the outcomes which were reported. If differences occur which are not adequately explained, or if studies do not report data which could reasonably be assumed to have been collected, we will consider such studies to be at high risk of reporting bias.

\section{Data synthesis}

Once heterogeneity is assessed, considering the $\mathrm{I}^{2}$ value and the magnitude and direction of effects, a decision will be made as to whether meta-analysis is appropriate. If it is, both random-effects and fixed-effect methods will be used in RevMan and the results compared. If the two methods give similar results, heterogeneity is not likely to be problematic and the random-effects results will be presented. If the results for the two methods differ, potential sources of heterogeneity will be investigated to determine which method is more appropriate. A preference will be given to using the more conservative option. If meta-analysis is deemed to be inappropriate, a structured narrative review will be written. In that case, forest plots will be generated if appropriate, but with the pooled estimate suppressed.

\section{Subgroup analysis and investigation of heterogeneity}

We will analyse the following subgroups.

1. Patients with metastatic cancer (TNM stage M1) versus patients with non-metastatic disease (TNM stage M0).

2. Patients with lymph node-negative versus node-positive prostate cancer.

3. Patients with Gleason scores of 6 or less (low risk), 7 (intermediate risk) and 8 or more (high risk).
4. Patients who entered the study with a history of no prior hormone therapy, hormone-dependent cancer, castrationresistant disease defined by rising PSA, and other castrationresistant disease.

5. Atrasentan versus zibotentan

\section{Sensitivity analysis}

We will perform the following sensitivity analyses, if $\mathrm{I}^{2}$ is greater than $50 \%$, to determine whether meta-analysis results are robust.

1. Studies will be assessed using the revised Cochrane risk of bias tool. Trials with a high risk of bias will be excluded.

2. Studies will be excluded if methodological quality or reliability could have been compromised due to issues other than bias. Specifically, we will exclude studies which allowed progression to be defined by rising PSA in the absence of clinical changes, or by measures of bone disease defined less stringently than recommended by the PSAWG2 (Scher 2008). If a proportion of patients are categorised as having disease progression, but any change in their condition is clinically insignificant, this could artificially reduce progression-free survival and time to progression.

3. Studies will be excluded if outcome data are missing from more than $20 \%$ of patients in either treatment or control group.

4. We will exclude conference proceedings and analyse only articles published in full.

\section{ACKNOWLEDGEMENTS}

We are grateful to the editorial team of the Prostatic Diseases and Urologic Cancers Review Group for their help and support. We would like to thank Dr Tim Grant of the Health Research Board Centre for Support and Training in Analysis and Research (CSTAR), University College Dublin, for his statistical advice. We thank Dr Steff Lewis of the Cochrane Statistical Methods Group for her guidance on ordinal data analysis.

\section{REFEREN CES}

\section{Additional references}

\section{Battistini 2006}

Battistini B, Berthiaume N, Kelland NF, Webb DJ, Kohan DE. Profile of past and current clinical trials involving endothelin receptor antagonists: the novel "-sentan" class of drug. Experimental Biology and Medicine 2006;231(6): 653-95.

\section{Benaim 2002}

Benaim EA, Pace CM, Lam PM, Roehrborn CG. Nadir prostate-specific antigen as a predictor of progression to androgen-independent prostate cancer. Urology 2002;59

(1):73-8. 
Carducci 2002

Carducci MA, Nelson JB, Bowling MK, Rogers T,

Eisenberger MA, Sinibaldi V, et al.Atrasentan, an endothelinreceptor antagonist for refractory adenocarcinomas: safety and pharmacokinetics. Journal of Clinical Oncology 2002; 20(8):2171-80

\section{Catalona 1984}

Catalona WJ. Prostate Cancer. Orlando: Grune and Stratton, 1984.

Dahut 2008

Dahut WL, Scripture C, Posadas E, Jain L, Gulley JL, Arlen $\mathrm{PM}$, et al.A phase II clinical trial of sorafenib in androgenindependent prostate cancer. Clinical Cancer Research 2008; 14(1):209-14.

de Bono 2010

de Bono JS, Logothetis CJ, Fizazi K, North S, Chu L, Chi KN. Abiraterone acetate plus low dose prednisone improves overall survival in patients with metastatic castration-resistant prostate cancer who have progressed after docetaxel-based chemotherapy: results of COU-AA301, a randomized double-blind placebo-controlled Phase III study. Annals of Oncology 2010;21(Supplement 8):viii3

\section{Eisenhauer 2009}

Eisenhauer EA, Therasse P, Bogaerts J, Schwartz LH, Sargent D, Ford R, et al.New response evaluation criteria in solid tumours: revised RECIST guideline (version 1.1). European Journal of Cancer 2009;45(2):228-47.

Higgins 2011

Higgins JPT, Green S (editors). Cochrane Handbook for Systematic Reviews of Interventions Version 5.1.0 [updated March 2011]. The Cochrane Collaboration, 2011. Available from www.cochrane-handbook.org (accessed 30 March 2011)

Ilic 2010

Ilic D, O'Connor D, Green S, Wilt TJ. Screening for prostate cancer. Cochrane Database of Systematic Reviews 2010, Issue 11. [DOI: 10.1002/14651858.CD004720.pub2]

\section{Jemal 2010}

Jemal A, Center MM, DeSantis C, Ward EM. Global patterns of cancer incidence and mortality rates and trends. Cancer Epidemiology, Biomarkers \& Prevention 2010;19(8): 1893-907.

Kirby 2001

Kirby RS, Christmas TJ, Brawer MK. Prostate Cancer. 2nd Edition. London: Mosby, 2001.

\section{Langenstroer 1993}

Langenstroer P, Tang R, Shapiro E, Divish B, Opgenorth T, Lepor H. Endothelin-1 in the human prostate: tissue levels, source of production and isometric tension studies. Journal of Urology 1993;150(2 Part 1):495-9.

Le Brun 1999

Le Brun G, Aubin P, Soliman H, Ropiquet F, Villette $\mathrm{JM}$, Berthon P, et al.Upregulation of endothelin 1 and its precursor by IL-1beta, TNF-alpha, and TGF-beta in the
PC3 human prostate cancer cell line. Cytokine 1999;11(2): $157-62$.

Nabhan 2009

Nabhan C, Lestingi TM, Galvez A, Tolzien K, Kelby SK, Tsarwhas D, et al.Erlotinib has moderate single-agent activity in chemotherapy-naive castration-resistant prostate cancer: final results of a phase II trial. Urology 2009;74(3): $665-71$.

National Library of Medicine (US) 2011 National Library of Medicine (US). ClinicalTrials.gov. Available from www.clinicaltrials.gov/ (accessed 8 January 2011).

\section{Nelson 2003}

Nelson J, Bagnato A, Battistini B, Nisen P. The endothelin axis: emerging role in cancer. Nature Reviews Cancer 2003; 3(2):110-6

\section{NICE 2008}

National Institute for Health and Clinical Excellence (UK). Prostate cancer:diagnosis and treatment. Available from http://www.nice.org.uk/nicemedia/live/11924/39687/ 39687.pdf (accessed 12 May 2011). London: National Institute for Health and Clinical Excellence, 2008.

Normanno 2009

Normanno N, Morabito A, De Luca A, Piccirillo MC, Gallo M, Maiello MR, et al.Target-based therapies in breast cancer:current status and future perspectives. EndocrineRelated Cancer 2009;16:675-702.

\section{Oefelein 2004}

Oefelein MG, Agarwal PK, Resnick MI. Survival of patients with hormone refractory prostate cancer in the prostate specific antigen era. The Journal of Urology 2004;171(4): $1525-8$.

\section{Ranson 2010}

Ranson M, Wilson RH, O'Sullivan JM, Maruoka M, Yamaguchi A, Cowan RA, et al.Pharmacokinetic and tolerability profile of once-daily zibotentan (ZD4054) in Japanese and Caucasian patients with hormoneresistant prostate cancer. International Journal of Clinical Pharmacology and Therapeutics 2010;48(11):708-17.

\section{Robey 1987}

Robey PG, Young MF, Flanders KC, Roche NS, Kondaiah P, Reddi AH, et al.Osteoblasts synthesize and respond to transforming growth factor-type beta (TGF-beta) in vitro. Journal of Cell Biology 1987;105(1):457-63.

Russo 2010

Russo A, Bronte G, Rizzo S, Fanale D, Di Gaudio F, Gebbia $\mathrm{N}$, et al.Anti-endothelin drugs in solid tumors. Expert Opinion on Emerging Drugs 2010;15(1):27-40.

\section{Schelman 2011}

Schelman WR, Liu G, Wilding G, Morris T, Phung D, Dreicer R. A phase I study of zibotentan (ZD4054) in patients with metastatic, castrate-resistant prostate cancer. Investigational New Drugs 2011;29(1):118-25. 
Scher 2004

Scher HI, Eisenberger M, D’Amico AV, Halabi S, Smal EJ, Morris M, et al.Eligibility and outcomes reporting guidelines for clinical trials for patients in the state of a rising prostate-specific antigen: recommendations from the Prostate-Specific Antigen Working Group. Journal of Clinical Oncology 2004; Vol. 22, issue 3:537-56.

\section{Scher 2008}

Scher HI, Halabi S, Tannock I, Morris M, Sternberg CN, Carducci MA, et al.Design and end points of clinical trials for patients with progressive prostate cancer and castrate levels of testosterone: recommendations of the Prostate Cancer Clinical Trials Working Group. Journal of Clinical Oncology 2008;26(7):1148-59.

Shelley 2006

Shelley M, Harrison C, Coles B, Staffurth J, Wilt TJ, Mason MD. Chemotherapy for hormone-refractory prostate cancer. Cochrane Database of Systematic Reviews 2006, Issue 4. [DOI: 10.1002/14651858.CD005247.pub2]

Tangen 2003

Tangen CM, Faulkner JR, Crawford ED, Thompson IM, Hirano D, Eisenberger M, et al.Ten-year survival in patients with metastatic prostate cancer. Clinical Prostate Cancer 2003;2(1):41-5.

Trump 2010

Trump DL, Payne H, Miller K, De Bono JS, Stephenson J,
Burris III, HA, et al.Phase I study of the specific endothelin A receptor antagonist zibotentan (ZD4054) combined with docetaxel in patients with metastatic castration-resistant prostate cancer: Assessment of efficacy, pain, and safety. Journal of Clinical Oncology 2010;28(No 15 (Supplement)): 4664.

Yanagisawa 1988

Yanagisawa M, Kurihara H, Kimura S, Tomobe Y, Kobayashi M, Mitsui Y, et al.A novel potent vasoconstrictor peptide produced by vascular endothelial cells. Nature 1988;332(6163):411-5.

Yin 2003

Yin JJ, Mohammad KS, Kakonen SM, Harris S, Wu-Wong JR, Wessale JL, et al.A causal role for endothelin-1 in the pathogenesis of osteoblastic bone metastases. Proceedings of the National Academy of Sciences of the United States of America 2003;100(19):10954-9.

Yuyama 2004

Yuyama H, Koakutsu A, Fujiyasu N, Fujimori A, Sato S, Shibasaki K, et al.Inhibitory effects of a selective endothelinA receptor antagonist YM598 on endothelin-1-induced potentiation of nociception in formalin-induced and prostate cancer-induced pain models in mice. Journal of Cardiovascular Pharmacology 2004;44 (Supplement 1): S479-82.

* Indicates the major publication for the study

\section{A P P E N D I C E S}

\section{Appendix I. RECIST criteria for tumour response to treatment}

The Response Evaluation Criteria in Solid Tumours (RECIST) criteria (Eisenhauer 2009) divide response to treatment into:

1. complete response, i.e. remission/disappearance of all target lesions';

2. partial response, i.e. at least $30 \%$ decrease in the sum of the longest diameter of target lesions;

3. progressive disease, i.e. at least $20 \%$ increase in the sum of the longest diameter of target lesions;

4. stable disease, i.e. neither sufficient shrinkage to qualify for partial response nor sufficient increase to qualify for progressive disease.

\section{H I S T O R Y}

Protocol first published: Issue 8, 2011 


\section{CONTRIBUTIONSOFAUTHORS}

Dr R Foley conceived, designed, coordinated and wrote the protocol. Dr J Keane provided a clinical perspective, contributed to writing the text and edited the protocol. Professor D Hollywood provided a clinical perspective and edited the protocol. Dr D O’Mathúna provided methodological advice and edited the protocol.

\section{DECLARATIONSOF INTEREST}

None declared.

\section{SOURCES OF SUPPORT}

\section{Internal sources}

- No sources of support supplied

\section{External sources}

- Health Research Board, Ireland.

Funding for Dr R Foley 